\title{
Gross Total Resection
}

National Cancer Institute

\section{Source}

National Cancer Institute. Gross Total Resection. NCI Thesaurus. Code C131672.

Surgical removal of an entire visible lesion, with no obvious lesion detected on postoperative evaluation; microscopic residual disease may be present. 